Pacific Journal of Mathematic 


\title{
CHARACTERIZATION OF COLLECTIVELY COMPACT SETS OF LINEAR OPERATORS
}

\author{
J. D. DePree and H. S. Klein
}

\begin{abstract}
The basic results in this paper show that each collectively compact set of linear operators can be viewed as an equicontinuous collection followed by a single compact operator. This observation not only gives insight into the character of collectively compact sets of linear operators, but also yields easier proofs of many of the results obtained by earlier workers in the field.
\end{abstract}

1. Factorizations of collectively compact operators. A fairly complete treatment, with applications, of collectively compact sets of linear operators is given in the recent book [1] by Anselone. Collectively compact sets of linear operators on normed linear spaces were originally studied by Anselone and Moore [2] in connection with approximate solutions of integral and operator equations.

The general properties of such sets of operators, again in normed linear spaces, were studied by Anselone and Palmer in [3] and [4]. Collectively compact sets of linear operators were studied in the more general setting of linear topological spaces by DePree and Higgins [5]. In the current work new characterizations are given for collectively compact sets of operators on a linear topological space.

We assume that $X$ and $Y$ are separated topological vector spaces and that $[X, Y]$ is the space of all continuous linear operators from $X$ to $Y$. For a collection $\mathscr{F} \leqq[X, Y]$ and $U$ a subset of $X$, let $\mathscr{F}(U)=\{T(x): x \in U, T \in \mathscr{F}\}$. For a set $\Omega$ with topology $\tau$, we adopt the notation $\langle\Omega, \tau\rangle$. For a set $\mathscr{N}$ of operators, we will be making statements of the following nature: Viewed as mappings between the unit ball of $Y^{*}$ endowed with its relative weak-star topology and $X^{*}$ equipped with norm topology, $\mathscr{N}$ is equicontinuous; we shall simply say that $\mathscr{N}:\left\langle Y^{*}\right.$, weak $\left.{ }^{*}\right\rangle \rightarrow\left\langle X^{*}\right.$, norm $\rangle$ is equicontinuous.

Following the work of DePree and Higgins [5], we make the following definition.

Definition 1.1. Let $X$ and $Y$ be separated topological vector spaces. Then $\mathscr{F} \subseteq[X, Y]$ is collectively compact if there exists a neighborhood $U$ of the origin in $X$ such that $\mathscr{F}(U)$ has compact closure in $Y$.

The easy proof of the following lemma belies its importance for 
the situation described in it is typical of collectively compact sets of operators; i.e., they can always be factored as in Theorem 1.3.

Lemma 1.2. Let $X, Y$, and $Z$ be separated topological vector spaces, $\mathscr{N} \subseteq[X, Z]$ an equicontinuous collection, and $K \in[Z, Y] a$ compact operator. Then the collection $K \mathscr{N}=\{K N: N \in \mathscr{N}\}$ is collectively compact.

Proof. Let $V$ be a 0-neighborhood in $Z$ such that $\overline{K(V)}$ is compact. Since the family $\mathscr{N}$ is equicontinuous, there is a 0 neighborhood $U$ in $X$ such that $\mathscr{N}(U) \subseteq V$. Thus $\overline{K \mathscr{N}(U)} \subseteq \overline{K(V)}$. It follows that $K \mathscr{N}$ is collectively compact.

THeOREM 1.3. Let $\mathscr{F} \subseteq[X, Y]$ be such that there exists a 0-neighborhood $U$ in $X$ with the closure of the balanced convex hull of $\mathscr{F}(U)$ compact in $Y$. Then there exist

(a) a Banach space $Z$,

(b) an equicontinuous collection $\mathscr{N} \subseteq[X, Z]$, and

(c) a compact operator $K \in[Z, Y]$

such that $\mathscr{F}=K N$.

Proof. The following proof is based upon the construction of an auxiliary normed space.

Let the set $C$ be the closure of the balanced convex hull of $\mathscr{F}(U)$ and $Z$ the span of $C$ in $Y$. Since $C$ is balanced and convex, $Z=\bigcup_{n=1}^{\infty} n C$ and $C$ is absorbing in $Z$. Hence $p$, the Minkowski functional of $C$, is defined on $Z$.

If $\langle Z, p\rangle$ denotes the set $Z$ endowed with the topology generated by $p$, then let $K:\langle Z, p\rangle \rightarrow Y$ be the natural injection which maps a point $z \in Z$ to the same point $z$ considered as an element of $Y$. $K$ is a compact operator since $C$, the unit ball of $Z$, is compact in $Y$. In particular, $K$ is continuous and the $p$-topology on $Z$ is stronger than the Hausdorff relative topology on $Z$. So $\langle Z, p\rangle$ is Hausdorff and $Z$ is a normed linear space.

Let $\left\{z_{n}\right\}$ be a Cauchy sequence in $\langle Z, p\rangle$. Since $\left\{z_{n}\right\}$ is a bounded subset of $\langle Z, p\rangle$ and $K$ is a compact operator, $\left\{K\left(z_{n}\right)\right\}$ is a Cauchy sequence with $\overline{\left\{K\left(z_{n}\right)\right\}}$ compact in $Y$. So there exists a $y \in Y$ such that $\lim _{n} K\left(z_{n}\right)=y$. For $\alpha>0$, choose $l$ such that $n, m \geqq l$ implies that $p\left(z_{n}-z_{m}\right) \leqq \alpha$. For these $n$ and $m, z_{n}-z_{m}$ is an element of $\alpha C$. In $Y, C$ is a closed set and $y=\lim _{n} z_{n}$. So $y-z_{m}$ is an element of $\alpha C$ for $m \geqq l$. We see that $y \in Z$ and that $p\left(y-z_{m}\right) \leqq \alpha$ for $m \geqq l$. It follows that $\langle Z, p\rangle$ is a Banach space.

Let $x \in X$ and $T \in \mathscr{F}$. Since 0 -neighborhoods are absorbing and 
$U$ is such a neighborhood in $X$, there exists an $\alpha>0$ such that $x \in \alpha U$. Hence, $T(x) \in \alpha C$ and $T(x) \in Z$. So let $\mathscr{N} \subseteq[X, Z]$ be defined as the collection $\mathscr{F}$ mapping $X$ to $\langle Z, p\rangle$. The collection $\mathscr{N}$ is equicontinuous since $\mathscr{N}(U)$ is a subset of the unit ball of $Z$. Obviously, $\mathscr{F}=K \mathscr{N}$.

Suppose $\mathscr{F} \subseteq[X, Y]$ satisfies the hypothesis of Theorem 2.3 and can be factored, $\mathscr{F}=K \mathscr{N}$, as above. The single compact operator $K$ has been the object of study for years. The next section shows that our knowledge about $K$ gives insight into the collection $\mathscr{F}$.

2. Characterizations of collectively compact operators defined on Banach spaces. Throughout this section, $X$ and $Y$ will be Banach spaces with closed unit balls $X_{1}$ and $Y_{1}$, respectively, $X^{*}$ and $X^{* *}$ will denote the first and second duals of $X$ with their usual norm topologies, and $[X, Y]$ will be the space of continuous linear operators from $X$ to $Y$ endowed with the uniform operator topology.

Note that $\mathscr{F} \subseteq[X, Y]$ is collectively compact if and only if $\mathscr{F}\left(F_{1}\right)$ has compact closure in $Y$.

Lemma 2.1. Let $\mathscr{F} \subseteq[X, Y] . \mathscr{F}$ is collectively compact if and only if there exist

(a) a Banach space $Z$,

(b) an equicontinuous collection $\mathscr{N} \leqq[X, Z]$, and

( c) a compact operator $K \in[Z, Y]$

such that $\mathscr{F}=K \mathscr{N}$.

Proof. Mazur's theorem [6, p. 416] states that if $\mathscr{F}\left(X_{1}\right)$ is relatively compact, then so is the balanced convex hull of $\mathscr{F}\left(X_{1}\right)$. Apply Lemma 1.2 and Theorem 1.3.

For $T \in[X, Y]$, let $T^{*} \in\left[Y^{*}, X^{*}\right]$ denote the adjoint of $T$. While Schauder's theorem implies that the adjoint of a compact operator is compact, the following example shows that this phenomenon has no generalization for collectively compact sets of operators. This example will also serve as an illustration for the results of the remainder of this paper.

EXAMPLE 2.2. Let $X=Y=l_{2}$ with the usual orthonormal basis $\left\{e_{k}: k=1,2, \cdots\right\}$. For each positive integer $n$, define $T_{n}$ by letting $T_{n}(x)=\left(x, e_{n}\right) e_{1}$. The set $\left\{T_{n}: n \geqq 1\right\}$ is collectively compact since $\mathrm{U}_{n} T_{n}\left(X_{1}\right)$ is a bounded subset of the finite dimensional subspace generated by $e_{1}$.

However, the collection of adjoints $\left\{T_{n}^{*}\right\}$ is not collectively compact 
since $T_{n}^{*}(x)=\left(x, e_{1}\right) e_{n}$ and $\mathrm{U}_{n} T_{n}^{*}\left(X_{1}^{*}\right)$ contains the orthonormal basis $\left\{e_{k}\right\}$.

Lemma 2.3. Let $\mathscr{F} \subseteq[X, Y]$. Then $\mathscr{F}^{*}=\left\{T^{*}: T \in \mathscr{F}\right\} \subseteq$ $\left[Y^{*}, X^{*}\right]$ is collectively compact if and only if there exists a Banach space $Z$, an equicontinuous collection $\mathscr{S} \subseteq[Z, Y]$, and a compact operator $K \in[X, Z]$ such that $\mathscr{F}=\mathscr{S} K=\{S K: S \in \mathscr{S}\}$.

Proof. Assume that $\mathscr{F}=\mathscr{S} K$, with $K$ a compact operator and $\mathscr{S}$ equicontinuous. The process of taking adjoints is an antihomomorphism which preserves operator norms. So if $\mathscr{F}=\mathscr{S} K$, then $\mathscr{S}^{*}=K^{*} \mathscr{S}^{*}$. If $\mathscr{S}$ is an equicontinuous (i.e., bounded) subset of $[Z, Y]$, then $\mathscr{S}^{*}$ is an equicontinuous subset of $\left[Y^{*}, Z^{*}\right]$. Lemma 2.1 implies that $\mathscr{F}^{*}$ is collectively compact.

Conversely, if $\mathscr{F}^{*}$ is collectively compact, there exists a Banach space $W$, an equicontinuous collection $\mathscr{P} \leqq\left[Y^{*}, W\right]$, and a compact operator $L \in\left[W, X^{*}\right]$ such that $\mathscr{F}^{*}=L \mathscr{P}$.

Let $J_{x}$ and $J_{y}$ denote the natural injections of $X$ into $X^{* *}$ and $Y$ into $Y^{* *}$, respectively. Note that $\mathscr{F}=J_{y}^{-1} \mathscr{F} * * J_{x}=\left(J_{y}^{-1} \mathscr{P} *\right)\left(L^{*} J_{x}\right)$. Let $K=L^{*} J_{x}$. Then $K$ is a compact operator mapping $X$ into $W^{*}$.

Since $J_{y}$ is an isometry and $\mathscr{P}^{*}$ is equicontinuous, it follows that $\mathscr{S}=J_{y}^{-1} \mathscr{P} *$ is an equicontinuous subset of $\left[W^{*}, Y\right]$ such that $\mathscr{S} K=\left(J_{y}^{-1} \mathscr{P}^{*}\right) K=\mathscr{F}$.

A description of the bounded weak-star topology of a Banach space $Y$ is given in [6, pages 427-430]. The feature of the bounded weak-star topology that will be of interest to us is the equivalence of parts (i) and (ii) of the following theorem.

Theorem 2.4. Let $Y$ be a Banach space. If $Y_{1}^{*}$ denotes the closed unit ball of $Y^{*}$, then a set $U \subseteq Y^{*}$ is a bounded weak-star neighborhood of 0 if and only if either one of the following are satisfied:

(i) For each $\alpha>0, \mathrm{U} \cap \alpha Y_{1}^{*}$ is a relative weak-star neighborhood of 0 in $\alpha Y_{1}^{*}$.

(ii) There exists a sequence $\left\{y_{n}\right\} \subseteq Y$ such that $\lim _{n}\left\|y_{n}\right\|=0$ and $\left\{y^{*} \in Y^{*}:\left|\left\langle y^{*}, y_{n}\right\rangle\right| \leqq 1\right.$ for each $\left.n\right\}$ is a subset of $U$.

Of course, statement (ii) may be rephrased in the form: There is a sequence $\left\{y_{n}\right\} \subseteq Y$ converging to 0 in norm such that the polar of $\left\{y_{n}\right\}$ is a subset of $U$.

THEOREM 2.5. For $\mathscr{F} \subseteq[X, Y]$, the following are equivalent:

(a) $\mathscr{F}$ is collectively compact.

(b) $\mathscr{F}^{*}:\left\langle Y_{1}^{*}\right.$, weak $\left.{ }^{*}\right\rangle \rightarrow\left\langle X^{*}\right.$, norm $\rangle$ is equicontinuous. 
(c) $\mathscr{F}^{*}:\left\langle Y_{1}^{*}\right.$, weak $\left.{ }^{*}\right\rangle \rightarrow\left\langle X^{*}\right.$, norm $\rangle$ is equicontinuous at the origin.

(d) $\left\{y^{*}:\left\|T^{*}\left(y^{*}\right)\right\| \leqq 1\right.$ for each $\left.T^{*} \in \mathscr{F}^{*}\right\}$ is a bounded weakstar neighborhood of 0 .

(e) There exists a sequence $\left\{y_{n}\right\} \subseteq Y$ such that $\left\|y_{n}\right\| \rightarrow 0$ and $\mathscr{F}\left(X_{1}\right)$ is a subset of the closure of the balanced convex hull of $\left\{y_{n}\right\}$.

Proof. (a) implies (b). If $\mathscr{F}$ is collectively compact, Lemma 2.1 implies that there exists a Banach space $Z$ and a factorization of $\mathscr{F}$, which, after taking adjoints, is of the form:

(1) $\mathscr{F}^{*}=\mathscr{N}^{*} K^{*}$.

(2) $K^{*}:\left\langle Y^{*}\right.$, norm $\rangle \rightarrow\left\langle Z^{*}\right.$, norm $\rangle$ is a compact operator.

(3) $\mathscr{N}^{*}:\left\langle Z^{*}\right.$, norm $\rangle \rightarrow\left\langle X^{*}\right.$, norm $\rangle$ is equicontinuous.

Now since $K^{*}$ is a compact operator, $K^{*}$ : $\left\langle Y_{1}^{*}\right.$, weak $\left.{ }^{*}\right\rangle \rightarrow\left\langle Z^{*}\right.$, norm $\rangle$ is continuous: It maps bounded nets which converge in the weakstar topology to weak-star convergent nets which are also totally bounded in the norm topology of $Z^{*}$. By (3), $\mathscr{N}^{*} K^{*}$ is an equicontinuous collection of mappings of $\left\langle Y_{1}^{*}\right.$, weak $\left.{ }^{*}\right\rangle$ into $\left\langle X^{*}\right.$, norm $\rangle$. We see that (b) follows immediately from (1).

(b) implies (c). This implication is obvious.

(c) implies (d). If the situation $\left\|T^{*}\left(y^{*}\right)\right\| \leqq r$ for each $T^{*} \in \mathscr{F}^{*}$ is abbreviated $\left\|\mathscr{F}^{*}\left(y^{*}\right)\right\| \leqq r$, then for any $\alpha>0$, (c) implies that $\left\{y^{*}:\left\|\mathscr{F}^{*}\left(y^{*}\right)\right\| \leqq 1 / \alpha\right\} \cap Y_{1}^{*}$ is a relative weak-star neighborhood of 0 in $Y_{1}^{*}$. Multiplication by $\alpha$ yields that $\left\{y^{*}:\left\|\mathscr{F}^{*}\left(y^{*}\right)\right\| \leqq 1\right\} \cap \alpha Y_{1}^{*}$ is a relative weak-star neighborhood of 0 in $\alpha Y_{1}^{*}$. Theorem 2.4, part (i), yields (d).

(d) implies (e). Statement (d) together with Theorem 2.4, part (ii), guarantee the existence of a sequence $\left\{y_{n}\right\} \subseteq Y$ such that $\left\|y_{n}\right\| \rightarrow 0$ and

(4) $\left\{y^{*}:\left|\left\langle y^{*}, y_{n}\right\rangle\right| \leqq 1\right.$ for each $\left.n\right\} \leqq\left\{y^{*}:\left\|\mathscr{F}^{*}\left(y^{*}\right)\right\| \leqq 1\right\}$. Now take polars in $Y$ of both of the above sets. By the Bipolar Theorem [7, p. 141], the polar of the left-hand side of (4) is the closure of the balanced convex hull of $\left\{y_{n}\right\}$. Since $\left\|\mathscr{F}^{*}\left(y^{*}\right)\right\| \leqq 1$, implies that $\left\|y^{*}\left(\mathscr{F}\left(X_{1}\right)\right)\right\| \leqq 1$, the polar of the right-hand of (4) contains $\mathscr{F}\left(X_{1}\right)$.

(e) implies (a). The set $\left\{y_{n}\right\}$ is compact. Therefore, the closure of the balanced convex hull of $\left\{y_{n}\right\}$ is also compact.

The following corollary was first proved by Palmer [8]. A new and simpler proof is given below.

CoRollary 2.6. Let $\mathscr{F} \subseteq[X, Y]$. If

(a) $F$ is collectively compact, and if 
(b) for each $y^{*} \in Y^{*}, \mathscr{F}^{*}\left(y^{*}\right)$ is totally bounded in the norm topology of $X^{*}$,

then $\mathscr{F}$ is totally bounded in $[X, Y]$.

Proof. By Theorem 2.5, (a) implies that if we consider $\mathscr{F}^{*}$ as a set of mappings between $\left\langle Y_{1}^{*}\right.$, weak $\left.{ }^{*}\right\rangle$ and $\left\langle X^{*}\right.$, norm $\rangle$, then $\mathscr{F}^{*}$ is equicontinuous with respect to these topologies. Since $\left\langle Y_{1}^{*}\right.$, weak $\left.{ }^{*}\right\rangle$ is a compact topological space, (b) together with the Ascoli theorem [7, p. 81] imply that the collection $\mathscr{F}^{*}$ is totally bounded in the topology of uniform convergence on $Y_{1}^{*}$, that is, in the uniform operator topology. Since the adjoint is an isometry between $[X, Y]$ and $\left[Y^{*}, X^{*}\right], \mathscr{F}$ is totally bounded in $[X, Y]$.

Corollary 2.7. If $\mathscr{N} \leqq[X, Y]$ is totally bounded and each member of $\mathscr{N}$ is a compact operator, then $\mathscr{N}$ is collectively compact.

Proof. If $\mathscr{N} \cong[X, Y]$ is totally bounded, so is $\mathscr{N}^{*} \cong\left[Y^{*}, X^{*}\right]$, i.e., $\mathscr{N}^{*}$ is totally bounded in the topology of uniform convergence on $Y_{1}^{*}$. Since each $T^{*} \in \mathscr{N}^{*}$ is a compact operator, each

$$
T^{*}:\left\langle Y_{1}^{*}, \text { weak }^{*}\right\rangle \longrightarrow\left\langle X^{*}, \text { norm }\right\rangle
$$

is continuous. Considered as a collection of mappings between the topological spaces of (5), $\mathscr{N}^{*}$ must be equicontinuous. By Theorem $2.5, \mathscr{N}$ is collectively compact.

In order to extend the range of application of Corollary 2.6, the following theorem is stated.

THEOREM 2.8. Let $\mathscr{S}=\left\{S_{n}: n \geqq 1\right\}$ be a sequence of bounded linear maps from $X$ to $Y$. Suppose there exists a collectively compact set $\left\{V_{n}: n \geqq 1\right\} \cong[X, Y]$ such that $\lim _{n \rightarrow \infty}\left\|S_{n}-V_{n}\right\|=0$. If $\mathscr{S}^{*}\left(y^{*}\right)$ is a totally bounded subset of $X^{*}$ for each $y^{*} \in Y^{*}$, then $\mathscr{S}$ is a totally bounded subset of $[X, Y]$.

Proof. Since $\lim _{n}\left\|S_{n}-V_{n}\right\|=0, \quad \lim _{n}\left\|S_{n}^{*}-V_{n}^{*}\right\|=0 . \quad$ Let $y^{*} \in Y^{*}$ and $\varepsilon>0$ be given. Choose an integer $N$ such that

$$
\left\|S_{n}^{*}\left(y^{*}\right)-V_{n}^{*}\left(y^{*}\right)\right\| \leqq \varepsilon / 3 \text { for } n \geqq N .
$$

$\mathscr{S}^{*}\left(y^{*}\right)$ is totally bounded and consequently has a finite $\varepsilon / 3$-net. The inequality (6) then implies that $\left\{V_{n}^{*}\left(y^{*}\right): n \geqq N\right\}$ has a finite $\varepsilon$-net. Since the excluded points are finite in number and $\varepsilon>0$ is arbitrary, the set $\left\{V_{n}^{*}\left(y^{*}\right): n \geqq 1\right\}$ is totally bounded. By Corollary 
2.6, $\left\{V_{n}: n \geqq 1\right\}$ is a totally bounded subset of $[X, Y]$. However, $\lim _{n}\left\|S_{n}-V_{n}\right\|=0$. By using an argument similar to the one above, it follows that $\mathscr{S}$ is a totally bounded subset of $[X, Y]$.

LEMma 2.9. Let $\mathscr{S} \subseteq[X, Y]$. If for each $x \in X, \mathscr{S}(x)$ is totally bounded in $Y$, then $\mathscr{S}^{*}:\left\langle Y_{1}^{*}\right.$, weak $\left.{ }^{*}\right\rangle \rightarrow\left\langle X^{*}\right.$, weak $\left.{ }^{*}\right\rangle$ is equicontinuous at the origin.

Proof. Let $x$ be any fixed element of $X$. Then

$$
W=\left\{x^{*}:\left|\left\langle x^{*}, x\right\rangle\right| \leqq 1\right\}
$$

is a neighborhood of 0 in the weak-star topology of $X^{*}$. In fact, the family of all such $W$ form a sub-basis of the neighborhood system of 0 for the weak-star topology. Therefore, it suffices to show that $Y_{1}^{*} \cap\left\{y^{*}: \mathscr{S}^{*}\left(y^{*}\right) \subseteq W\right\}=Y_{1}^{*} \cap\left\{y^{*}:\left|\left\langle\mathscr{S}^{*}\left(y^{*}\right), x\right\rangle\right| \leqq 1\right\}=$ $Y_{1}^{*} \cap\left\{y^{*}:\left|\left\langle y^{*}, \mathscr{S}(x)\right\rangle\right| \leqq 1\right\}$ is a neighborhood of 0 in the relative weak-star topology on $Y_{1}^{*}$.

Let $\left\{y_{1}, y_{2}, \cdots, y_{n}\right\}$ be a $1 / 2$-net for $\mathscr{S}(x)$. Consider $V=$ $\left\{y^{*}:\left|\left\langle y^{*}, y_{i}\right\rangle\right| \leqq 1 / 2, \quad 1 \leqq i \leqq n\right\}$. If $y^{*} \in V \cap Y_{1}^{*}$, and $y \in \mathscr{S}(x)$, choose $j$ such that $\left\|y-y_{j}\right\| \leqq 1 / 2$. Then $\left|\left\langle y^{*}, y\right\rangle \leqq\right|\left\langle y^{*}, y_{j}\right\rangle \mid+$ $\left|\left\langle y^{*}, y-y_{j}\right\rangle\right| \leqq 1 / 2+\left\|y^{*}\right\|\left\|y-y_{j}\right\| \leqq 1$ since $y^{*} \in Y_{1}^{*}$. So $V \cap Y_{1}^{*} \cong$ $Y_{1}^{*} \cap\left\{y^{*}: \mathscr{S}^{*}\left(y^{*}\right) \subseteq W\right\}$. It follows that

$$
\mathscr{S}^{*}:\left\langle Y_{1}^{*}, \text { weak }^{*}\right\rangle \longrightarrow\left\langle X^{*}, \text { weak }^{*}\right\rangle
$$

is equicontinuous at the origin.

Theorem 2.10. Let $X, Y$, and $Z$ be Banach spaces and let $\mathscr{F} \subseteq[X, Z]$ be collectively compact. For $\mathscr{S} \subseteq[Z, Y]$, suppose $\mathscr{S}(z)$ is totally bounded in $Y$ for each $Z \in Z$. Then $\mathscr{S} \mathscr{F}=$ $\{S T: S \in \mathscr{S}, T \in \mathscr{F}\}$ is collectively compact.

Proof. Since $\mathscr{S}(z)$ is bounded for each $z \in Z$ and $Z$ is complete, there exists a constant $m$ such that $\|S\| \leqq m$ for each $S \in \mathscr{S}$. If $U$ is any 0-neighborhood in the norm topology of $X^{*}$, choose, by Theorem 2.5, a weak-star neighborhood $W$ of 0 in $Z^{*}$ such that $\mathscr{F}^{*}\left(W \cap Z_{1}^{*}\right) \subseteq(1 / m) U$. Lemma 2.9 guarantees that there exists a weak-star neighborhood $V$ of 0 in $Y^{*}$ such that $\mathscr{S}^{*}\left(V \cap Y_{1}^{*}\right) \subseteq m W$. So $(1 / m) \mathscr{S}^{*}\left(V \cap Y_{1}^{*}\right) \subseteq W \cap(1 / m) \mathscr{S}^{*}\left(Y_{1}^{*}\right) \subseteq W \cap Z_{1}^{*}$. It follows that $\mathscr{F}^{*}\left((1 / m) \mathscr{S}^{*}\left(V \cap Y_{1}^{*}\right)\right) \subseteq(1 / m) U$ and that $(\mathscr{S} \mathscr{F})^{*}:\left\langle Y_{1}^{*}\right.$, weak $\left.{ }^{*}\right\rangle \rightarrow$ $\left\langle X^{*}\right.$, norm $\rangle$ is equicontinuous at the origin. Theorem 2.5 implies that the set $\mathscr{S} \mathscr{F}$ is collectively compact.

EXAMPLE 2.2 continued. Let $C=\left\{x:\left\|T_{n}(x)\right\| \leqq 1\right.$ for each $\left.n\right\}$. 
Then $C=\left\{x:\left|\left(x, e_{n}\right)\right| \leqq 1\right.$ for each $\left.n\right\}$ and $C$ is the polar of $\left\{e_{n}\right\} . \quad C$ is not a bounded weak* neighborhood of 0 , since if it were, the Bipolar Theorem would then imply that the orthonormal basis $\left\{e_{n}\right\}$ is a compact subset of $l_{2}$. Since $l_{2}$ is a Hilbert space, one can view $\left\{T_{n}\right\}$ as the adjoint of the collection $\left\{T_{n}^{*}\right\}$. Theorem 2.5, part (d), implies that $\left\{T_{n}^{*}\right\} \subseteq\left[l_{2}, l_{2}\right]$ is not collectively compact. In particular, an explicit calculation of the adjoints is unnecessary in determining whether or not $\left\{T_{n}^{*}\right\}$ is collectively compact. The next theorem shows that it is unnecessary to calculate the adjoints even when the operators involved are acting on arbitrary Banach spaces.

ThEOREm 2.11. For $\mathscr{F} \leqq[X, Y]$, the following are equivalent:

(a) $\mathscr{F}^{*}\left[Y^{*}, X^{*}\right]$ is collectively compact.

(b) $\mathscr{F}:\left\langle X_{1}\right.$, weak topology $\rangle \rightarrow\langle Y$, norm $\rangle$ is equicontinuous.

(c) $\mathscr{F}:\left\langle X_{1}\right.$, weak topology $\rangle \rightarrow\langle Y$, norm $\rangle$ is equicontinuous at the origin.

(d) There exists a sequence $\left\{x_{n}^{*}\right\} \subseteq X^{*}$ such that $\left\|x_{n}^{*}\right\| \rightarrow 0$ and $\left\{x \in X:\left|\left\langle x_{n}^{*}, x\right\rangle\right| \leqq 1\right.$ for each $\left.n\right\} \subseteq\{x:\|\mathscr{F}(x)\| \leqq 1\}$.

( e ) There exists a sequence $\left\{x_{n}^{*}\right\} \subseteq X^{*}$ such that $\left\|x_{n}^{*}\right\| \rightarrow 0$ and $\mathscr{F}^{*}\left(Y_{1}^{*}\right)$ is a subset of the closure of the balanced convex hull of $\left\{x_{n}^{*}\right\}$.

Proof. The equivalence of (a) and (e) follows from Theorem 2.5.

The polar of the closure of the balanced convex hull of $\left\{x_{n}^{*}\right\}$ is $\left\{x:\left|\left\langle x_{n}^{*}, x\right\rangle\right| \leqq 1, n \geqq 1\right\}$. Also, the polar of $\mathscr{F}^{*}\left(Y_{1}^{*}\right)$ is $\{x:\|\mathscr{F}(x)\| \leqq$ 1) since $\left|\left\langle\mathscr{F}^{*}\left(Y_{1}^{*}\right), x\right\rangle\right| \leqq 1$ if and only if $\left|\left\langle Y_{1}^{*}, \mathscr{F}(x)\right\rangle\right| \leqq 1$. The equivalence of (d) and (e) follows from these two observations.

(a) implies (b). By Lemma 2.3, there exists a Banach space $Z$, a compact operator $K \in[X, Z]$, and an equicontinuous collection $\mathscr{S} \subseteq$ $[Z, Y]$ such that $\mathscr{F}=\mathscr{S} K$. Since $K$ is a compact operator

$K:\left\langle X_{1}\right.$, weak $\rangle \longrightarrow\langle Z$, norm $\rangle$ is continuous .

Moreover, $\mathscr{S}:\langle Z$, norm $\rangle \rightarrow\langle Y$, norm $\rangle$ is equicontinuous. Hence, $\mathscr{F}:\left\langle X_{1}\right.$, weak $\rangle \rightarrow\langle Y$, norm $\rangle$ is equicontinuous.

(b) implies (c). This implication is obvious.

(c) implies (a). By Theorem 2.5, it suffices to show that $\mathscr{F}^{* *}:\left\langle X_{1}^{* *}\right.$, weak $\left.{ }^{*}\right\rangle \rightarrow\left\langle Y^{* *}\right.$, norm $\rangle$ is equicontinuous at the origin. If $J$ denotes the natural injection of $X$ into $X^{* *}$, (c) implies that

$$
\mathscr{F}^{* *}:\left\langle J\left(X_{1}\right), \text { weak }^{*}\right\rangle \longrightarrow\left\langle Y^{* *}, \text { norm }\right\rangle
$$

is equicontinuous at the origin.

Let $V$ be a 0 -neighborhood in the norm topology of $Y^{* *}$. Choose a 0 -neighborhood $U$ such that $\bar{U} \subseteq V$, where the bar denotes 
closure in the norm topology of $Y^{* *}$. By (8), choose $W$, a 0 neighborhood in the weak-star topology of $X^{* *}$, such that

$$
\mathscr{F}^{* *}\left(J\left(X_{1}\right) \cap W\right) \subseteq U \text {. }
$$

Let $T^{* *}$ be an element of $\mathscr{F}^{* *}$ and $x^{* *} \in X_{1}^{* *} \cap W$. Since $J\left(X_{1}\right)$ is weak-star dense in $X_{1}^{* *}$, it is possible to choose a net $\left\{x_{\alpha}\right\} \subseteq X_{1}$ such that the weak-star limit of $\left\{J\left(X_{\alpha}\right)\right\}$ is $x^{* *} .\left\{J\left(x_{\alpha}\right)\right\}$ is eventually in $W$ since $x^{* *} \in W$. Therefore, $\left\{T^{* *}\left(J\left(x_{\alpha}\right)\right)\right\}$ is eventually in $U$, by (8). Since $T^{* *}$ is a compact operator, $\left\|T^{* *}\left(x^{* *}\right)-T^{* *}\left(J\left(x_{\alpha}\right)\right)\right\| \rightarrow 0$. Hence $T^{* *}\left(x^{* *}\right) \in \bar{U} \subseteq V$. So in addition to (8),

$$
\mathscr{F}^{* *}\left(X_{1}^{* *} \cap W\right) \subseteq V \text {. }
$$

In order to indicate how some previous results in the theory of collectively compact operators follow from our results, we prove the following lemma.

LEMMA 2.12. Let $L \subseteq[X, Y]$ be bounded in the uniform operator topology. The following are equivalent:

(a) $L:\left\langle X_{1}\right.$, weak $\rangle \rightarrow\langle Y$, norm $\rangle$ is equicontinuous at the origin.

(b) For each $\varepsilon>0$, there exists a subspace $X(\varepsilon)$ of finite codimension in $X$ such that the restrictions of operators in $L$ to $X(\varepsilon)$ have operator norms no greater than $\varepsilon$.

Proof. Let the bound on $\mathscr{L} \subseteq[X, Y]$ be $M$, i.e., $\|T\| \leqq M$ for each $T \in \mathscr{L}$.

(a) implies (b). Let $\varepsilon>0$. By (a), there exists a finite set $\left\{x_{1}^{*}, \cdots, x_{p}^{*}\right\} \leqq X^{*}$ such that $\left\{x:\left|\left\langle x_{i}^{*}, x\right\rangle\right| \leqq 1, \quad 1 \leqq i \leqq p\right\} \cap X_{1} \subseteq$ $\{x:\|\mathscr{L}(x)\| \leqq \varepsilon\}$. Let $X(\varepsilon)=\left\{x:\left\langle x_{i}^{*}, x\right\rangle=0,1 \leqq i \leqq p\right\}$. Then (b) follows since $X(\varepsilon) \cap X_{1} \subseteq\{x:\|\mathscr{L}(x)\| \leqq \varepsilon\}$.

(b) implies (a). Let $\varepsilon$ and $X(\varepsilon)$ be given. If $T \in \mathscr{L}$, then the operator norms of the restrictions of $T$ to $X(\varepsilon)$ and $\overline{X(\varepsilon)}$ are the same. Consequently, we may assume that $X(\varepsilon)$ is a closed subspace of $X$.

Choose linearly independent $\left\{x_{1}, \cdots, x_{p}\right\} \subseteq X$ such that $\left\|x_{i}\right\|=1$ for each $i$ and $X=X(\varepsilon) \oplus \operatorname{Span}\left\{x_{i}\right\}$. Since for each $j, X(\varepsilon) \oplus$ Span $\left\{x_{1}, \cdots, x_{j-1}, x_{j+1}, \cdots, x_{p}\right\}$ is a closed subspace which does not contain $x_{j}$, there exists $\left\{x_{i}^{*}: 1 \leqq i \leqq p\right\} \subseteq X^{*}$ such that $x_{i}^{*}(X(\varepsilon))=0$, $1 \leqq i \leqq p$, and $x_{i}^{*}\left(x_{j}\right)=\delta_{i, j}, 1 \leqq i, j \leqq p$. Consider the weak open set

$$
W=\left\{x: \sum_{i=1}^{p}\left|\left\langle x_{i}^{*}, x\right\rangle\right| \leqq \min \{\varepsilon, 1\}\right\}
$$

If $x \in W \cap X_{1}$, then $x$ has the representation 


$$
x=x_{\varepsilon} \oplus \sum_{i=1}^{p}\left\langle x_{i}^{*}, x\right\rangle x_{i}
$$

with $x_{\varepsilon} \in X(\varepsilon)$. Since $\left\|x_{i}\right\|=1$,

$$
\left\|x_{\varepsilon}\right\| \leqq\|x\|+\sum_{i=1}^{p}\left|\left\langle x_{i}^{*}, x\right\rangle\right| \leqq 2 \text {. }
$$

Then for $T \in L,\left\|T\left(X_{\varepsilon}\right)\right\| \leqq 2 \varepsilon$ and

$$
\|T(x)\| \leqq 2 \varepsilon+\sum_{i=1}^{p}\left|\left\langle x_{i}^{*}, x\right\rangle\right|\left\|T\left(x_{i}\right)\right\| \leqq 2 \varepsilon+M \varepsilon .
$$

We have shown that for any $\varepsilon>0,\{x:\|\mathscr{L}(x)\| \leqq 2 \varepsilon+M \varepsilon\} \cap X_{1}$ is a relative weak neighborhood of the origin. Hence, statement (a) follows.

Finally, in view of Theorem 2.11, one obtains the result of Palmer [8] that for the collection $\mathscr{L}$ above, $\mathscr{L}^{*}$ is collectively compact if and only if condition (b) of the above lemma is satisfied.

\section{REFERENCES}

1. P. M. Anselone, Collectively Compact Operator Approximation Theory and Applications to Integral Operators, Prentice-Hall, 1971.

2. P. M. Anselone and R. H. Moore, Approximate solutions of integral and operator equations, J. Math. Anal. Appl., 9 (1964), 268-277.

3. P. M. Anselone and T. W. Palmer, Collectively compact sets of linear operators, Pacific J. Math., 25 (1968), 417-422.

4. - Spectral analysis of collectively compact, strongly convergent operator sequences, Pacific J. Math., 25 (1968), 423-431.

5. J. D. DePree and J. A. Higgins, Collectively compact sets of linear operators, Math. Z., 115 (1970), 366-370.

6. N. Dunford and J. T. Schwartz, Linear Operators, Part I. New York: Interscience, 1958.

7. J. L. Kelly and I. Namioka, Linear Topological Spaces, Princeton: Van Nostrand, 1963.

8. T. W. Palmer, Totally bounded sets of precompact linear operators, Amer. Math. Soc., 29 No. 1, (1969), 101-106.

Received April 4, 1973.

New Mexico State University 


\section{PACIFIC JOURNAL OF MATHEMATICS}

EDITORS

RICHARD ARENS (Managing Editor)

University of California

Los Angeles, Calıfornia 90024

R. A. Beaumont

University of Washington

Seattle, Washington 98105
J. DugundJI

Department of Mathematics

University of Southern California

Los Angeles, California 90007

D. Gilbarg and J. Milgram

Stanford University

Stanford, California 94305

\section{ASSOCIATE EDITORS}
E. F. BECKENBACH
B. H. NeumanN
F. WOLF
K. YOSHIDA

\section{SUPPORTING INSTITUTIONS}

UNIVERSITY OF BRITISH COLUMBIA

CALIFORNIA INSTITUTE OF TECHNOLOGY

UNIVERSITY OF CALIFORNIA

MONTANA STATE UNIVERSITY

UNIVERSITY OF NEVADA

NEW MEXICO STATE UNIVERSITY

OREGON STATE UNIVERSITY

UNIVERSITY OF OREGON

OSAKA UNIVERSITY
UNIVERSITY OF SOUTHERN CALIFORNIA

STANFORD UNIVERSITY

UNIVERSITY OF TOKYO

UNIVERSITY OF UTAH

WASHINGTON STATE UNIVERSITY

UNIVERSITY OF WASHINGTON

AMERICAN MATHEMATICAL SOCIETY NAVAL WEAPONS CENTER 


\section{Pacific Journal of Mathematics}

\section{Vol. 55, No. $1 \quad$ September, 1974}

Robert Lee Anderson, Continuous spectra of a singular symmetric

differential operator on a Hilbert space of vector-valued functions . . . $\quad 1$

Michael James Cambern, The isometries of $L^{p}(X, K) \ldots \ldots \ldots \ldots \ldots . . \ldots$

R. H. Cameron and David Arne Storvick, Two related integrals over spaces of continuous functions ................................

Gary Theodore Chartrand and Albert David Polimeni, Ramsey theory and

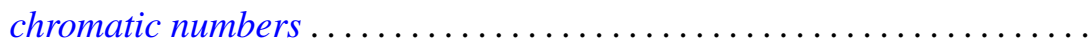

John Deryck De Pree and Harry Scott Klein, Characterization of collectively compact sets of linear operators ...................

John Deryck De Pree and Harry Scott Klein, Semi-groups and collectively compact sets of linear operators ....................... 55

George Epstein and Alfred Horn, Chain based lattices.............. 65

Paul Erdős and Ernst Gabor Straus, On the irrationality of certain series . . 85

Zdeněk Frolík, Measurable uniform spaces................... 93

Stephen Michael Gagola, Jr., Characters fully ramified over a normal

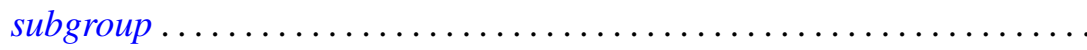

Frank Larkin Gilfeather, Operator valued roots of abelian analytic

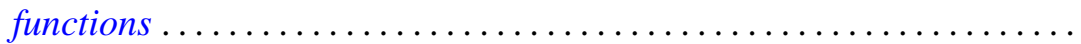

D. S. Goel, A. S. B. Holland, Cyril Nasim and B. N. Sahney, Best approximation by a saturation class of polynomial operators

James Secord Howland, Puiseux series for resonances at an embedded

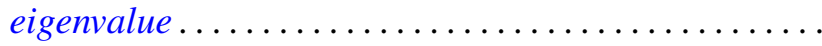

David Jacobson, Linear GCD equations .................

P. H. Karvellas, A note on compact semirings which are multiplicative

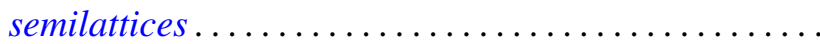

Allan Morton Krall, Stieltjes differential-boundary operators. II . .

D. G. Larman, On the inner aperture and intersections of convex sets

S. N. Mukhopadhyay, On the regularity of the $P^{n}$-integral and its application to summable trigonometric series ....... .

Dwight Webster Read, On $(J, M, m)$-extensions of Boolean algebras ....

David Francis Rearick, Multiplicativity-preserving arithmetic power series.

Indranand Sinha, Characteristic ideals in group algebras

Charles Thomas Tucker, II, Homomorphisms of Riesz spaces . . .

Kunio Yamagata, The exchange property and direct sums of indecomposable injective modules. 